\title{
Three-dimensional information modeling in the preservation of objects of cultural heritage
}

\author{
Tatiana Tsitman* and Kseniia Proshunina \\ ASUACE, Department ADR, 414056, st. Tatishcheva, Russia
}

\begin{abstract}
Currently, the project process is actively introducing a new approach to BIM technologies, implying comprehensive design. The advantage of BIM technologies allows monitoring the object at all steps of the design process. To conduct and compile cartograms of defects in complex scientific research it is necessary to use modern BIM technologies. The construction of a three-dimensional model helps to track defects and deformities in the process and makes it possible to carry out repair and restoration measures with greater efficiency. Restoration activities with BIM design involves the sequential implementation of activities that include: comprehensive scientific research, field surveys, engineering and geological research, and much more.

Full automation of information about the object under study will make it easier to work on the restoration of the monument and will provide an opportunity for making accurate and competent decisions. To do this, it is necessary to structure the information, highlighting the most reliable data for the periods of exploitation, materials, design features of the cultural heritage site.
\end{abstract}

\section{Introduction}

Three-dimensional information modeling is a new approach to the erection, equipping and operation of a building. BIM technologies become a tool that allows us to reproduce more complex architectural and engineering solutions in the design documentation in more detail before the construction starts, and to monitor the operation of the building in the future.

In European countries and the United States, this approach is already in use and is in demand. The model allows not only to carry out the design, but also to track further work at the construction, restoration, repair and restoration stages. International experience highlights the peculiarities of using BIM technologies taking into account the design of objects, such as: construction monitoring, creation of an information model for operation, creation of interactive three-dimensional applications.

\footnotetext{
* Corresponding author: taortsi@gmail.com
} 
For our country, the use of three-dimensional information modeling is an innovation and the introduction of such an approach to the construction sphere of activity is just beginning. For the environment historical and architectural system of the cities of the Russian Federation in the conditions of the development of modern architecture, an integrated approach with the introduction of three-dimensional information modeling technologies for the preservation of cultural heritage objects is especially topical.

With the advent of BIM technologies, it is possible to form a new direction in the approach to the restoration process, which allows not only to give an idea of the threedimensional model, but also to determine the interrelated nature of the work of structures and materials in different historical stages of the building's existence.

\section{Main Part}

The basis of the BIM methodology for restoration activities of the cultural heritage site can be laid down a project strategy, which includes the following stages of work:

1) input data:

- comprehensive scientific research;

- Archival-digital calculations;

- Comparative analogue selection;

- formation of information on time slices;

2) information processing:

- $\quad$ input of structural parameters of structural and other elements;

- $\quad$ Entering material specifications;

- $\quad$ Defining the structure of layers;

- Creation of the library elements and modules;

3) three-dimensional model:

- control of the restoration process;

- monitoring of processes occurring during the operation of a cultural heritage site:

- control of regular repair and restoration measures.

Proper identification of the input data is the most significant event and has a great importance in later reconstruction-rastavratsionnyh events. Before starting the virtual model, the structure of the final project is initially formed, saturated with information, supported by a number of studies obtained during the field information work, performed with the help of the latest technical support: a laser $3 \mathrm{~d}$ scanner; Gps equipment; unmanned vehicles, etc.

Filling occurs in the course of archival-digital calculations obtained during the photogrammetric analysis, which allows you to restore the size of photos. In cases of fragmentary or actual loss of the cultural heritage object, the restoration of the dimensions can be carried out according to the preserved historical photographs by means of specialized software.

When a fragmentary or actual loss of an object of cultural heritage is reconstructed, in the absence of archival material or historical images of the object under study, a comparative analogue selection is carried out characterized by a relatively close time of building construction and the materials used at a given time, identical stylistic solution of the object, other buildings, executed by the same author of the building, and other information that can reveal in the course of the selection the expected losses of the object under study.

For the full volume of three-dimensional modeling to preserve the historical image of the formation of the cultural heritage object, the ability to output information on temporal sections of all transformations of the object under study is a prerequisite for the 
development of the information model. Such a method makes it possible to trace the process of changes from the initial historical appearance to the final contemporary appearance. The process of virtual reconstruction is carried out with possible intermediate fixations, namely: fragmented and detailed.

For the processing of information, the systematization and archiving of input data is carried out. Table lists are created from the received input data resources for the file format in which the information of the file itself and additional information about the data stored in them are stored simultaneously, which are hidden from the user in the normal content browsing mode. Electronic spreadsheets represent text descriptions and are characterized by a level of detail in which the information graphic model and its information component, including information about the model, material, place of application, etc., are developed. In addition, the tables contain direct links for further virtual modeling and visualization work.

All files represented by a set of spreadsheet lists with input parameters represent a common metafile system. Important in the course of systematization of information is the definition of the structure of layers in chronology, such programming will allow to trace the process of reconstruction and all changes, which occurred with the object of cultural heritage from the beginning of construction to the present and in the reverse order by means of the process of "layers of reconstruction" laid down by the programs, supported by BIM technologies. Mutual substitution of detail in the further course of virtual simulation will trace the historical architectural design at various stages of history, which will make it possible to adopt the most suitable and competent option for the solution when carrying out restoration activities and writing an object into the existing historical and cultural environment.

The creation of a three-dimensional information model takes considerable space of information storage. For ease of use and reducing the weight of stored information, it is necessary to create modules that are formed into library elements, which can be stored for convenience on portable data carriers.

After the systematization and processing of the received resources into a metafile and possible parallel modeling, the completed process of the three-dimensional model is executed. This stage is marked by a verifiable simulation, excluding possible errors that can occur when organizing the joint work of all sections of engineering solutions.

The reconstruction project for the BIM approach is the most beneficial in terms of design. The three-dimensional model is interconnected not only with the projections of twodimensional drawings, it is documented according to materials and individual models, engineering solutions, allowing in aggregate to assess the performance of all structural elements, control the restoration process, control the processes occurring during the operation of the cultural heritage site, as well as control of the next repair and restoration activities.

The resulting three-dimensional model of the building continues to work for a long time, it allows to make changes and to monitor the building during its operation. Thus, the model will be the initial data of control and management of the object. BIM technologies are implemented with the help of a set of software and hardware, which allows to organize the joint work of all interested parties. When creating a detailed information model, the input data must be as accurate as possible, therefore the devices used must meet high requirements.

Carrying out field studies is a laborious and responsible process in the course of conducting pre-project work. The information received is the necessary basis for the organization of restoration and restoration activities, so architects and engineers pay special attention to this process. 
Surface solution of facades of monuments-architecture can represent a complex decorative art decoration, saturated with elements of architectural stucco, which will significantly increase the time for the execution of measurement drawings when performing measurements in the traditional way, by means of roulettes and plumb. At the same time, the human factor is taken into account with the possible deviations of the sizes from the real object, which in the future will entail deviations in the change in the costs of the restoration work [1].

Currently, the technology for production and processing of information in the conduct of field studies significantly advanced. Architects and restorers need the use of innovative equipment, since new technologies will maximize the time-cost in the implementation of restoration and reconstruction documentation of cultural heritage sites. Modern technical facilities allow you to perform volumetric reading of information from the surfaces of an object to within a millimeter and create three-dimensional maps of objects that can then be studied at any angle in 3D modeling. Such possibilities are represented by a digital laser 3d scanner.

With the help of a ground-based laser $3 \mathrm{~d}$ scanner, input data for restoration projects with virtual forecasting of further design stages are provided according to the BIM technology concept.

The principle of the laser $3 \mathrm{~d}$ scanner is based on the use of a laser range finder and a field PC. The scanner sends a laser beam to the surface of the scanned object, stumbling on the road to an obstacle, the beam returns, and the scanner generates up to a million dimensional points per second and has a 360-degree rotation angle relative to the scanner. The resulting slice from the point cloud is processed and surprisingly detailed threedimensional images are created afterwards.

When working with existing objects, laser 3D scanning can be used for the following purposes:

- research of objects of historical and cultural heritage, namely: three-dimensional scanning of the surface of facades, including decorative and artistic design (Figure 1);

- Research of structures of buildings and structures: detection of deformations, fixation of applicable solutions of nodes of structural elements;

- research of engineering networks with creation of spatial models of communications;

- converting the received surveys into a three-dimensional volume model as input for further work with the information model of the building.

- $\quad$ linking the captured model to the local coordinate system;

- control of the construction site during the re-scanning, when using the design of the BIM information modeling of the building, it is possible to create overlapping slots from the point cloud of the erected construction site to the working drawings of the design documentation and, when comparing, record the deviations recorded in the executive drawings. 


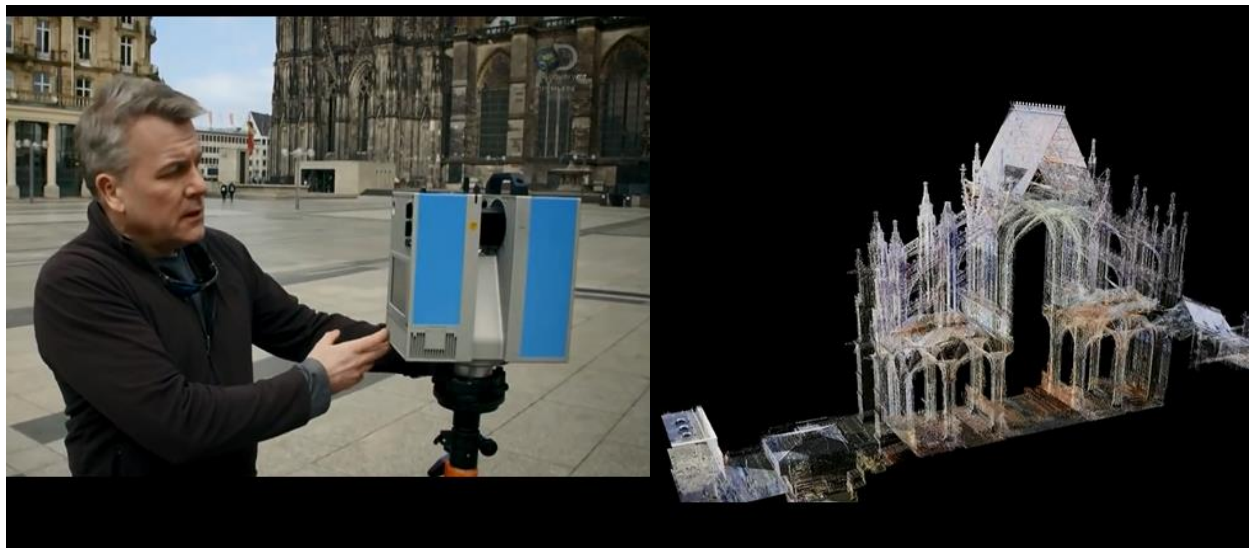

Fig. 1. Digital laser 3d scanner Discovery. Exploding history. Secrets of the medieval cathedrals [2].

Laser 3D scanning is a high-precision device that allows to carry out actual documentation in the process of construction and restoration and reconstruction activities. The capabilities of this equipment allow you to obtain the information of interest immediately in a three-dimensional image and use it to create a BIM information model of a building.

Unmanned equipment is a remotely controlled aircraft and has now received multiple applications in many areas, including construction. In addition to one of the important purposes of compact drones - the compilation of topographic maps of the terrain by means of aerial photography, new ways of using this technical device are added. When a technical device for photographic equipment and special equipment, with GPS software installed, are placed on an unmanned drone, a panorama of the historical and cultural environment and the object under investigation is created. Later, based on simple photography and software, a three-dimensional information model is created, the result is comparable to using a laser $3 \mathrm{~d}$ scanner. The use of unmanned equipment greatly saves time and costs for inspecting objects, in comparison with traditional methods of research (Fig. 2,3).

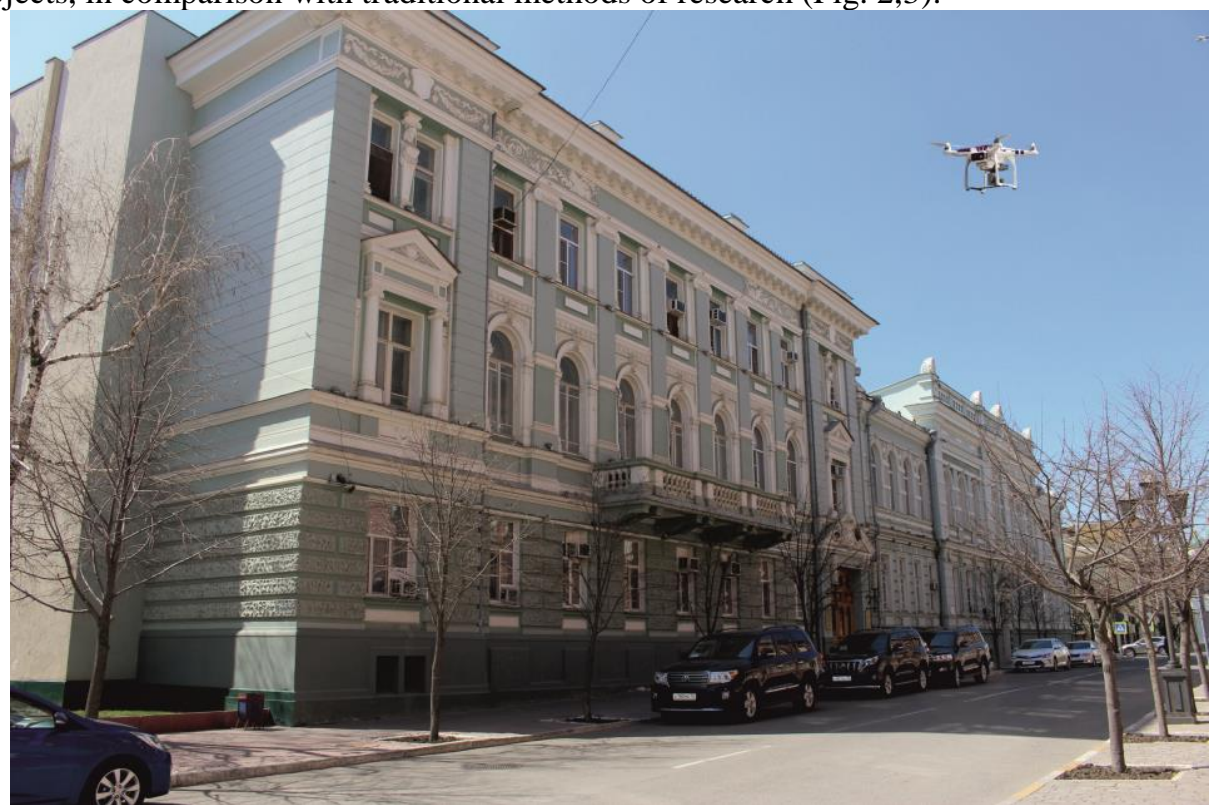

Fig. 2. Inspection of a cultural heritage site using unmanned equipment - a drone. 


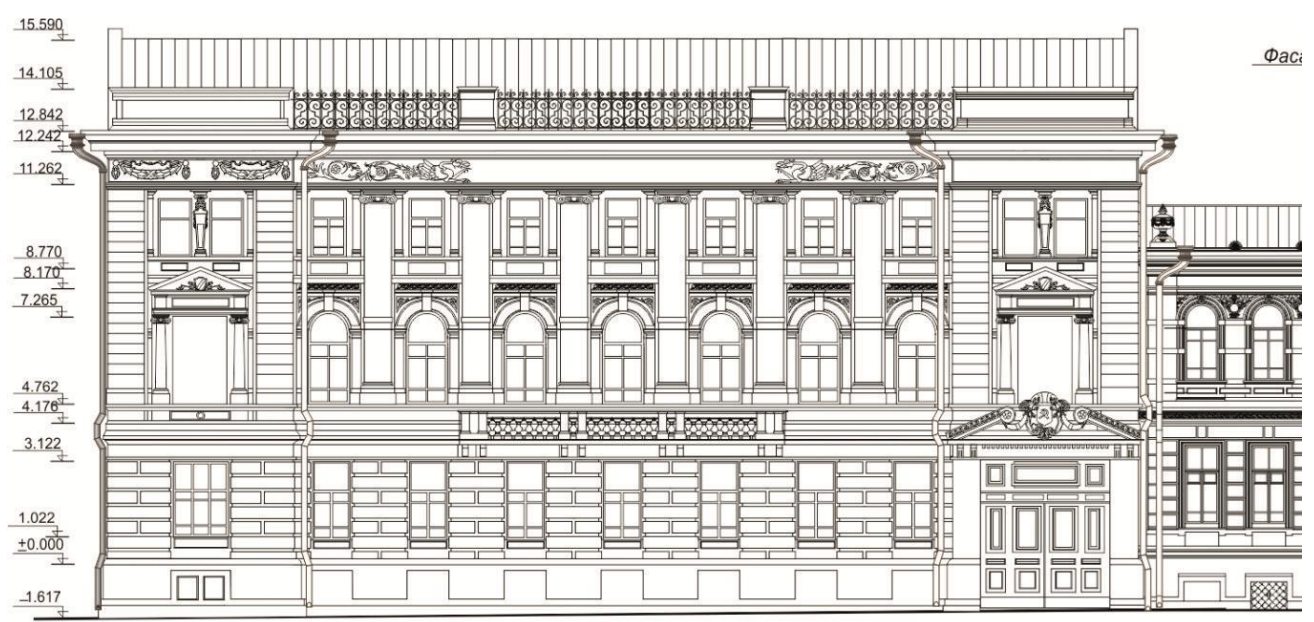

Fig. 3. Facades along Sovetskaya Street

Technological innovations also touched upon the determination of the physical and mechanical properties of masonry. As is known, porous stones can contain moisture inside the rock, which affects the strength characteristics of the material and, in general, the structural element. Therefore, in the most important areas of structural elements, regular inspection and study of the strength characteristics of the stone is necessary.

European restorers, when examining the Cologne Cathedral, eliminating the problem with the structural elements of arch buttresses made of soft limestone, carried out research using drilling equipment with a sensitive tip and with a connection to a personal computer that gives an idea of the rock density at the sites of the sections of the structures studied (Fig. 4)

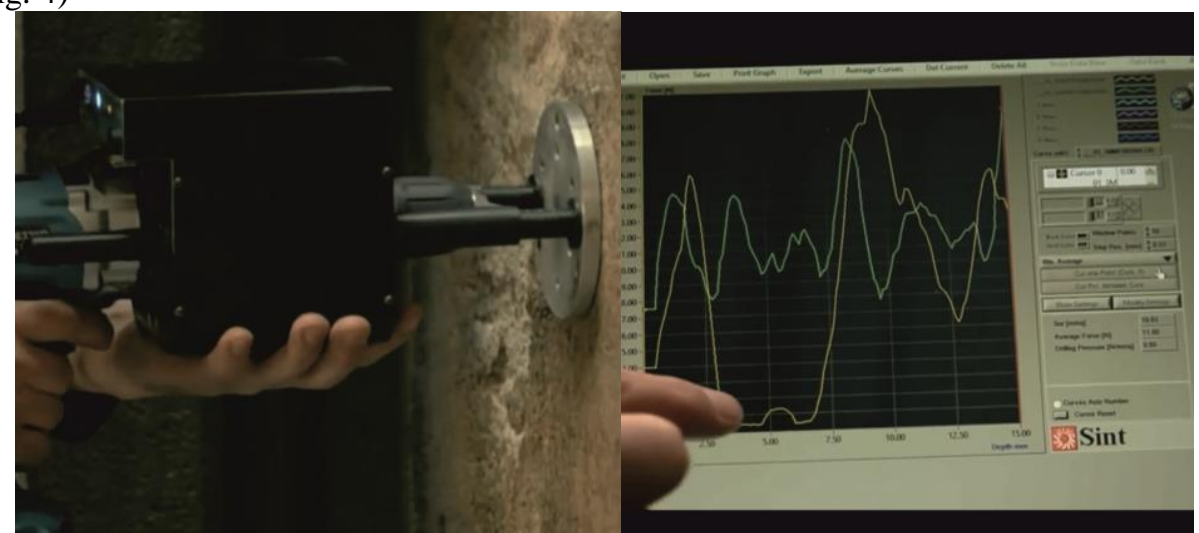

Fig. 4. Inspection for the strength of masonry.Discovery. Exploding history. Secrets of the medieval cathedrals [2].

Personal computer measures the resistance of the stone by using a dedicated software determines and damaged areas. According to the transferred indications of resistance measurements, a graph, showing the measurements in the form of a vector diagram, in which the take-off of the graph marks the stout sections of the masonry, the fall of the schedule - loose areas, which subsequently leads to conclusions on the implementation of measures to strengthen the stone and exclude repeated similar damage.

Every year modern measuring instruments are improved and transformed in their manufacturability. Instruments and sensors with high levels of signal processing are indispensable developments in the collection of data in emergency situations. Such 
measuring devices are used for different purposes and have a personal technological component, so they can get individual implementation in one or another area of use. The information model of the building created by BIM provides for control over the processes occurring during the operation of the construction object or the restored cultural heritage object [3]. In the case of objects of cultural heritage, deformation of structures can occur from various factors or their combined effect, for example: subsidence of foundations, resulting in the displacement of horizontal structures; deflections of floor beams and other deformations $[4,5]$. Particularly relevant is the control of cultural heritage sites, located in seismic regions, where, under the influence of geological underground activity, there can be significant changes in the structural body of the building and at a critical threshold, entailing consequences up to the collapse. Therefore, it is important to perform test measurements of activity and fix the state of the building's structures in order to be able to prevent current deformations and, in the end, to prevent possible catastrophic consequences. So for the monitoring of the building, devices and motion sensors are used, allowing measurements of the position of the structural elements to be measured and in the early stages to produce a potential detection of deformations.

Devices are placed floor by floor, in certain points of the building, and even the slightest fluctuations of the whole structure are captured. The sensors have a connection to the computer into which the instrument readings are transmitted. By monitoring the object for a certain period of time, statistical summaries are displayed with the indications of these devices, allows us to understand, what deformations in the investigated cultural heritage object can occur with insignificant seismic activity or with strong tremors (Fig.5).

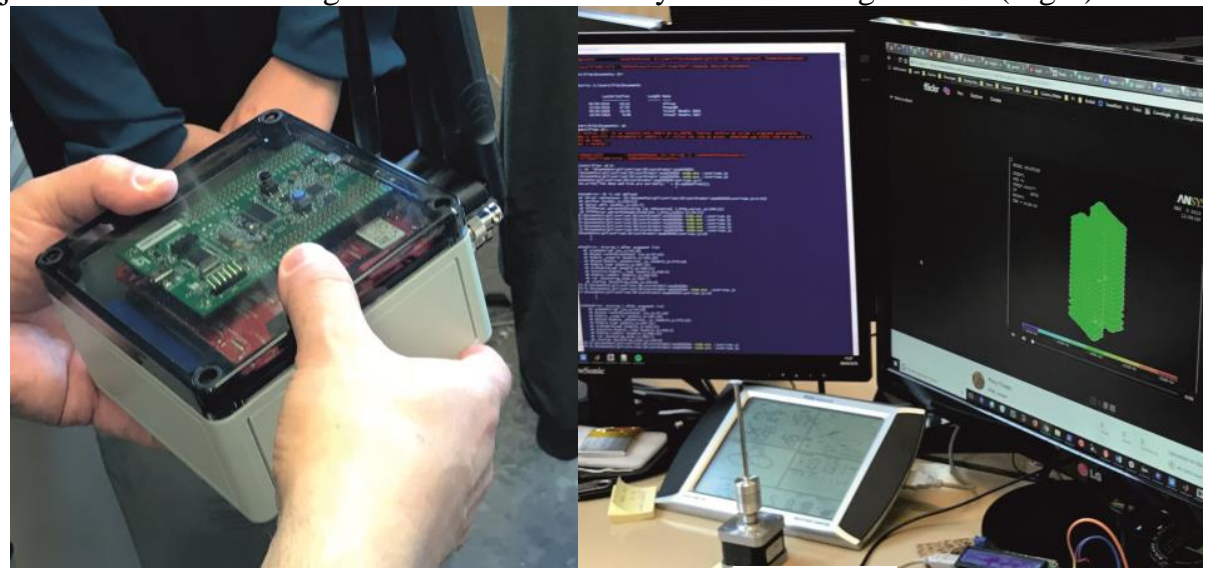

Fig. 5. Monitoring of deviations detected by motion sensors. Politechnical University of Madrid, Faculty of technological engineering.

Devices and building monitoring sensors are designed for the monitoring BIM information model of the building during operation, and are a modern necessity, since the constructions, with the course of time dilapidated, bearing capacity is reduced, and with regular seismic loads, significant structural damage to the building can occur.

BIM technologies have a number of advantages: geometric modeling, layering, the ability to control the model at any stage, etc. Using adaptive and parametric objects greatly facilitates the work process. The technique of layer reconstruction lays visualization capabilities that are close to historical reality, which allows us to make well-founded design and restoration solutions.

For the long-term preservation of architectural monuments, it is necessary to solve many problems related to various aspects of the reconstruction and restoration of architectural heritage sites. The existing changes in the environment, in the dynamics of the city's development, in modern requirements for architecture and restoration create 
prerequisites for in-depth study and detailed study of architectural and restoration concepts [6]. BIM technology approach will allow making decisions aimed at restoring the historical and artistic significance of architectural monuments.

To preserve the heritage in the conditions of the development of modern architecture, a comprehensive scientific approach to work with objects of cultural heritage is a necessary requirement. This approach includes:

- $\quad$ town planning solutions

- environmentally visual landscape solutions, to reveal the panoramic perspectives of the historical architectural and spatial environment

- architectural solutions of newly implemented capital construction projects in the historical and cultural environment

- $\quad$ engineering solutions for the preservation of existing architectural monuments

The totality of all decisions will create the basis for the competent preservation of the heritage of the architecture of the historic city center, it will allow for the systematic control of building, to produce a reorganization historic neighborhoods, upgrade environment while preserving the historic town status. In addition, work on carrying out engineering and restoration activities with objects of cultural heritage is of an individual nature. So the climate, geological characteristics, the availability of groundwater and the behavior of building materials in a particular environment are unique for each object of architecture. At the same time, the study of the examples in question will reveal certain patterns of the problematic restoration process.

Architectural losses of parts and parts of the building are restored bit by bit, based on the BIM technology with specific dating, during which the building was conducted, questions of the authenticity of building materials and methods of erecting architectural monuments are posed.

\section{Reference}

1. O.I. Prutsin Architectural and historical environment [Text], (Textbook for high schools-M: Institute of Art Restoration, 2004)

2. Discovery. Exploding history. Secrets of the medieval cathedrals [Electronic resource]: URL: https://www.youtube.com/watch?v=F0WlGgqf28Q

3. V.P. Knyazeva Ecology. Fundamentals of Restoration [Text] (Textbook. - M: Architecture-C, 2005)

4. E.M. Pashkin Engineering Geology [Text], (Textbook .- M .: Architecture - S, 2005.)

5. G.T. Popov, L.Y. Burak Technical examination of residential buildings of the old building [Text], (Leningrad: Stroyizdat, 1986.)

6. Y.V. Raninsky Monuments of architecture and town planning [Text]: A manual for architectural and building specialties], (M.: Higher School, 1988.) 